\title{
A unified cooperative model for environmental costs in supply chains: the Shapley value for the linear case
}

\author{
Francesco Ciardiello ${ }^{1} \cdot$ Andrea Genovese $^{1} \cdot$ Andrew Simpson $^{1}$
}

Published online: 28 August 2018

(c) The Author(s) 2018

\begin{abstract}
This paper provides a normative framework based on cooperative game theory aimed at studying the problem of pollution responsibility allocation across multi-tier supply chains. The model is further developed with reference to the case of a linear supply chain, by using three responsibility principles (namely: Upstream, Downstream and Local Responsibility). Allocation rules are derived; also, desirable properties in terms of fairness, efficiency and transparency are introduced, in order to characterize such rules. Furthermore, a stability concept for efficient allocations is formulated. An example of a possible application of the introduced cost allocation rules is provided.
\end{abstract}

Keywords Supply chains · Pollution responsibility allocation · Game theory · Shapley value

\section{Introduction}

The promotion of environmental sustainability has become crucial in the implementation and day-to-day functioning of complex and global supply networks. A growing number of large multi-national enterprises are implementing more stringent environmental practices; this has also an impact on small and medium-sized enterprises (SMEs) which are often involved in their supply networks (UN Global 2011).

The promotion of tighter environmental standards also implies the adoption of benchmarking approaches for comparing the sustainability performances of supply chains against industry standards. Within this context, Life Cycle Assessment (LCA) methodologies allow the estimation of cumulative environmental impacts across the entire supply chain and against a wide range of indicators, adopting a full product life cycle perspective. Within the current context of growing environmental sustainability, performing an allocation of environmental impacts to the different actors in the supply chain, with the aim of understanding which of them should be deemed responsible for polluting activities and related taxes or abatement costs, is a relevant and timely issue, if proper mitigation measures need to be implemented.

The interest in the topic of environmental pollution responsibility started due to the need to establish mechanisms to perform pollution burden sharing actions across countries (Zhou and

Andrea Genovese

a.genovese@sheffield.ac.uk

1 Sheffield University Management School, University of Sheffield, Conduit Road, Sheffield S10 1FL, UK 
Wang 2016); the related literature has then evolved in order to address allocation problems at different levels. According to an analysis of the current literature (see, for instance, Zhou and Wang 2016), most of the available approaches such problems at a macro-level; the application of pollution responsibilities approaches to contemporary multi-tier and multi-stakeholders supply chains is often overlooked.

This paper aims at addressing this research gap, by providing a normative framework based on cooperative game theory aimed at studying the problem of pollution responsibility allocation across multi-tier supply chains.

The paper is organised as follows. After a review of the literature concerned with pollution responsibility allocation, and the identification of relevant gaps (Sect. 2), the paper presents a cooperative game theoretical responsibility model for pollution allocation (Sect. 3). In Sect. 4, the paper details the generic model in the case of a linear supply chain. In Sect. 5, a practical application of the introduced cost allocation rules is provided; finally, some conclusions are drawn.

\section{Literature review}

The problem of associating pollution responsibilities with economic actors traces its roots in the international trade literature (Leontief and Ford 1970; Proops et al. 1993; Wyckoff and Roop 1994; Imura and Moriguchi 1995; Subak 1995; Bosquet 2000; Korhonen 2002; Bastianoni et al. 2004; Lenzen and Murray 2010). While the interest in the topic started because of the need to establish mechanisms to perform pollution burden sharing actions across countries, the related literature has then evolved in order to address allocation problems at different levels. These include the decomposition of national objectives into regional ones and the distribution of environmental pollution responsibility across partner firms in a given supply chain.

One of the fundamental research questions in the field of environmental pollution responsibility has been represented by the allocation principle to be followed. Scholars have developed different allocation criteria that can be mainly categorized into fairness- and efficiencyinspired principles. The concept of "fairness" is generally linked to equitable distribution issues (Rose 1990); the one of "efficiency" is more related to the minimization of costs related to abatement and mitigation measures. Many emissions allocation methods have been proposed. According to the extensive review performed by Zhou and Wang (2016), these can be classified into multiple categories. One of them is the so-called indicator approach. Through the development of specific indicators, pollution responsibilities, targets or permits are worked out in relation to these measures. Another stream of studies is devoted to the use of optimization approaches, which is based on mathematical programming frameworks for pollution allocation, which try to minimize costs for pollution abatements. For example, Ridgley (1996) combed the use of composite indicators and an optimization method for deriving suitable pollution responsibility allocations at a country level. This rapid scan of the extant literature, coherently with findings from Zhou and Wang (2016), highlights that most of the developed approaches deal with the pollution responsibility problem at a macro-level. Indeed, most of the methodologies tackle the problem at a country or regional level.

While some firm-level approaches can be retrieved, the supply chain perspective has been, so far, largely ignored, with the few available approaches being characterized by very simple and naïve models, mainly dealing with dyadic configurations; the application of pollution responsibilities approaches to contemporary multi-tier and multi-stakeholders supply chains is often overlooked. 
Pollution allocation mechanisms often include interactions involving multiple actors. As such, pollution allocation mechanisms may be represented in a very effective way through approaches based on Game Theory. As stated by Zhou and Wang (2016), game theoretic approaches seem to be naturally suited for this purpose. Such allocations result from equilibria among different parties' interactions or from values, which may satisfy enjoyable properties imposed by a central authority. Compared to the traditional approaches for pollution costs allocation, game theoretic approaches can be deemed as more sophisticated and less immediate. The use of game theoretic approaches is underexploited, especially when dealing with complex and multi-tier supply chains. Concepts from cooperative game theory may assume a normative value, i.e. a value that is imposed by an external authority.

Chander and Tulkens (1995) and Filar and Gaertner (1997) developed the first adaptation of Game Theory to the problem in order to investigate the allocation of GHGs emission reductions quota among countries. A similar approach, at an international level, was developed by Eyckmans and Tulkens (2003), while Germain and Steenberghe (2003) utilized a dynamic game framework. Viguier et al. (2006) deployed a two-level game for solving a similar allocation problem. At a firm level, MacKenzie et al. (2008, 2009) utilized, respectively, rank-order contests and incomplete information games for allocating pollution allowances. Chung et al. (2013) utilized dynamic games to evaluate the response of companies to environmental pollution taxes in a spatially distributed supply chain. Ren et al. (2015) proposed a Stackelberg game for studying $\mathrm{CO} 2$ reduction targets in a very simple dyadic supply chain.

Surprisingly, the development of normative approaches to pollution cost allocation is less extensive. A practical applications of the Shapley value approach was developed by Liao et al. (2015) for allocating emission allowances across energy producers in Shanghai.

\subsection{Contribution of the paper}

The next section presents a unified cooperative game theoretical approach to pollution responsibility allocation in a multi-tier supply chain. In Sect. 4, the paper details the generic model in the case of a linear supply chain, by selecting three different responsibility principles (namely: Upstream, Downstream and Local Responsibility). Also, fairness, efficiency and transparency properties are introduced, as desirable characteristics for allocation rules; furthermore, a stability concept for efficient allocations is formulated. In particular, here we present the Shapley value for this unified cooperative game theoretical responsibility model. In doing this, we find interesting connections between the so-called river problem introduced by Dong et al. (2012) in the pollution games literature and our framework. The Shapley allocation satisfies many of the mentioned desirable properties.

In Sect. 5, a practical application of the introduced cost allocation rules is provided, with the reference to the context of dairy supply chains. Finally, some conclusions are drawn.

\section{Cooperative responsibility models for environmental supply chains}

Consider a supply chain of a given product. Such a supply chain can be seen as a set $N$ of companies engaged, at various stages, in the manufacturing of the product, through sourcing of raw materials, sub-components manufacturing, assembly activities; such companies can be indexed in a given order. A set of processes $P_{i}$ (with $\left|P_{i}\right| \leq|N|-1$ ) can be associated with each company $i$. In this context, a process $(i, j)$ represents the production of goods by company $i$ and the supplying of such goods to company $j$ (with $i \neq j$ ) (as shown in Fig. 1). 
Fig. 1 Structure of a supply chain network

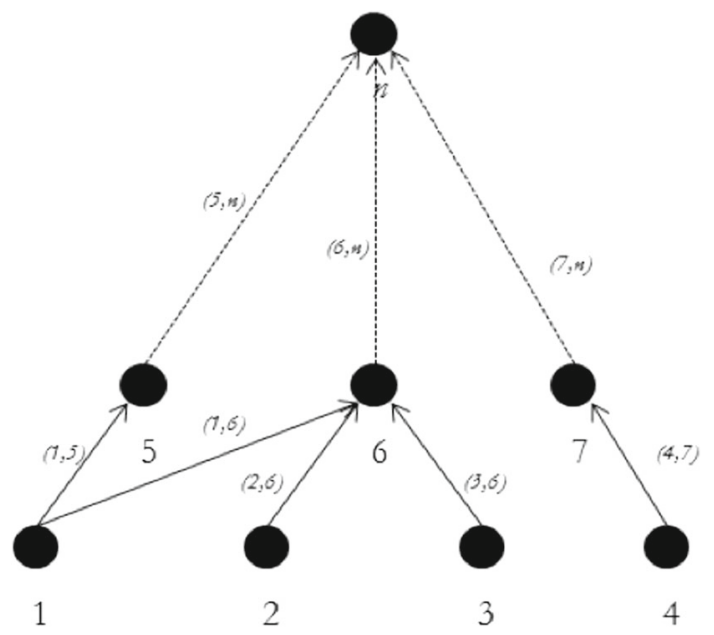

As such, $P_{i} \subseteq P$, where $P=\bigcup_{i=1 \ldots n} P_{i}$. The set $P_{i}$ might be empty and this implies that company $i$ is not supplying goods or services to any supply chain partner. Conversely, the set $P_{i}$ might be coinciding with the whole set of processes (i.e., $P$ ); this means that company $i$ is the only supplier in the considered network. Also, each process $(i, j)$ has an environmental cost that is denoted as $c_{i j}$. Let $C$ be the set of all environmental costs. Therefore, we build a mathematical framework as follows:

$$
(N, P, C)
$$

In order to enhance this framework with the concept of environmental responsibility, it can be useful to introduce a $|N| \times|P|$ responsibility matrix $B=\left(B_{i, p}\right)$ where the row index $i$ represents companies and the column index $p$ represents processes. Significantly, $b_{i p}=1$ if company $i$ is responsible (from an environmental point of view) for process $p, b_{i p}=0$ otherwise. Let $B_{i}$ be the set of the processes for which company $i$ is responsible from an environmental point of view, that is $B_{i}=\left\{p \in P \mid b_{i p}=1\right\}$. It is important to recall that the environmental responsibility of a process may not depend on the physical location of the process; in other words, $b_{i p}$ can be equal to 1 even if process does not involve physically company $i$ (in other words, if the company $i$ is not involved as a supplier in this process, or, alternatively, if the production of goods involved in the process does not happen at company $i$ premises). Therefore, the framework (1) can be rewritten as $(N, P, B, C)$, where $B$ is the responsibility matrix. The quantity $\sum_{p \in B_{i}} c_{p}$ can be interpreted as an intuitive cost allocation for company $i$, summing up all the environmental costs of processes $p$ for which company $i$ is deemed responsible. A coalition responsibility set can be defined as $B_{S}=\cup_{i \in S} B_{i}$; then, the social cost function for each sub-group of companies $S \subseteq N$ can be defined as follows:

$$
v(S)=\sum_{p \in B_{s}} c_{p}
$$

The sum $v(S)$ in the formula (2) represents the environmental cost of all the processes for which at least one company belonging to the set $\mathrm{S}$ is responsible. Moreover, each cost is counted only once as different companies may be responsible for the same process. ${ }^{1}$ Being

\footnotetext{
1 The environmental cost function can be also defined as $v(S)=\sum_{s \in S} \sum_{(i, j) \in B_{S} / \sqcup_{t=1}^{s-1} B_{t}} c_{i j}$ in an equivalent
way.
} 
$N$, by definition, a finite set, $v: 2^{N} \rightarrow R$ is a function which associates a real value with each subset of $N$. Following a classical definition, $(N, v)$ represents a cooperative game with a characteristic function. $N$ also represents the set of players; each subset of the set of players is called a coalition; $v$ is called the characteristic function of the game.

In this case, the characteristic function $v$ is defined through the above-introduced elements $P, B$ and $C$, therefore leading to the following model:

$$
G=(N, P, B, C, v)
$$

Such cooperative games are defined in terms of a characteristic function, which specifies the outcomes that each coalition can achieve for itself. Outcomes are specified in terms of the total utility/cost that a coalition can get and distribute across its members. By assuming the formation of a grand coalition, the main aim of such games is the definition of a solution concept, which allocates costs among each player from $N$.

In a supply chain context, it can be fair assuming that companies cooperate (by forming a grand coalition) in order to coordinate the activities of the production system. This way, they will incur in different individual production costs because of initial binding agreements among partners; also, they will face costs related to the mitigation of the environmental impacts of their production activities. Cooperative game theory can provide solutions to allocate environmental pollution impacts across each member of the production system, by allowing transferable payments among companies.

In this study, the main aim is to identify a vector $x=\left(x_{i}\right) \in R_{+}^{|N|}$ representing a solution to the cooperative model (2), which assigns the cost $x_{i}$ to each company $i$.

An allocation is responsible-compatible when, if $B_{i}$ is empty, then $\mathrm{x}_{\mathrm{i}}$ is null. In the following, we will always refer to allocations which respect the responsible-compatible principle.

An allocation is efficient if the sum of all cost-allocations, i.e. $\sum_{\mathrm{i}=1}^{|\mathrm{N}|} x_{i}$, is equal to the sum of all costs, i.e. $\sum_{i=1}^{|N|} \sum_{j=1}^{|N|} c_{i j} .^{2}$

\subsection{Desirable properties for cost allocation rules}

Cost allocation rules should respect some desirable properties that have been described in the environmental pollution responsibility literature. In the following, we list and discuss each of these properties; we remark that some of these have been already listed in Gopalakrishnan et al. (2016), while and some of them are novel.

Property 1: Equivalence If two companies $i$ and $j$ are responsible for the same processes, then they must have the same cost allocation. Therefore, if $B_{i}=B_{j}$ then $x_{i}=x_{j}$ must be satisfied for any $i$ and $j \in N$.

Property 2: Equal sharing of extra pollution This property provides fairness to the allocation of extra costs that might arise as the result of additional polluting activities (that could happen over time). If there is an increase in total pollution costs, it is required that companies responsible for this increase should be equally affected by extra burdens.

As a preliminary definition, it can be stated that $C^{\prime} \geq C$ if and only if $c_{i j}{ }^{\prime} \geq c_{i j}$ for any $i$, $j \in N$. We say that $C^{\prime}>C$ if $C^{\prime} \geq C$ and there exists a process $(i, j) \in P$ such that $c_{i j}{ }^{\prime}>c_{i j}$ Let $G=(N, P, B, C, v), G^{\prime}=\left(N, P, B, C^{\prime}, v\right)$ be the two associated cooperative models where $\mathrm{C}$ and $C^{\prime}$ are two sets of environmental costs with $C^{\prime} \geq C$. If $i, j \in N$ are responsible

$\overline{2 \text { We will denote an allocation } x_{i} \text { of the cooperative model } G \text { by } x(G)}=\left(x_{i}(G)\right) \in R_{+}^{|N|}$. 
for any process $p$, that is $b_{i, p}=b_{j, p}=1$ where $p \in P$ is characterized by $c_{p}{ }^{\prime}>c_{p}$. Then the following equality $x_{i}\left(G^{\prime}\right)-x_{i}(G)=x_{j}\left(G^{\prime}\right)-x_{j}(G)$ must be satisfied.

Property 3: No free riding The equal sharing of extra pollution property (introduced above as Property 2) does not guarantee the efficiency of such net cost increase re-allocation. In other words, the property does not require that the sum of extra charges for responsible companies is equal to the net increase of the pollution costs across the supply chain. As such, Property 3 provides an additional efficiency requirement to the above-mentioned situation. This property requires that if the total pollution costs increase, but, at the same time, for some firms the pollution of the processes they are responsible for remains unchanged, the allocation for these firms should remain the same.

Let $G=(N, P, B, C, v)$ and $G^{\prime}=\left(N, P, B, C^{\prime}, v\right)$ be the two associated cooperative models where $\mathrm{C}, C^{\prime}$ are two sets of environmental costs with $C^{\prime} \geq C$. Let $i \in N$; suppose that $c_{p}^{\prime}=c_{p}$ for each process $p$ satisfying $b_{i, p}=1$. Then, the equality $x_{i}\left(G^{\prime}\right)=x_{i}(G)$ must be satisfied.

Property 4: Process independence Suppose that a single process $p$ increases its pollution cost $c_{p}$; as a consequence, cost allocations across the supply chain will change. In other words, cost allocations might be affected by the new cost distribution over the set of all processes even if just one single process increases its cost. If this does not happen, it means that the variation of cost allocations solely depends on the process whose cost has increased; then, we say that cost allocations are process independent. As a consequence, this property implies that the firms can transparently relate their cost variations to the processes with increased costs; as such, they may make an official grievance against firms involved in those more costly processes.

Let $G=(N, P, B, C, v), G^{\prime}=\left(N, P, B, C^{\prime}, v\right), \tilde{G}=(N, P, B, \tilde{C}, v), \tilde{G}^{\prime}=(N$, $\left.P, B, \tilde{C}^{\prime}, v\right)$ be the cooperative models associated to the cost structures $C, C^{\prime}, \tilde{C}, \tilde{C}^{\prime}$.We assume that $C^{\prime} \geq C, \tilde{C}^{\prime} \geq \tilde{C}$ and $c_{p}^{\prime}=c_{p}, \tilde{c}_{p}^{\prime}=\tilde{c}_{p}$ for $p \in M \backslash\{q\}$. We assume that $\tilde{c}_{q}^{\prime}=c_{q}^{\prime}$ and $c_{q}=\tilde{\mathrm{c}}_{q}$. Then, $x_{i}\left(G^{\prime}\right)-x_{i}(G)=x_{i}\left(\tilde{G}^{\prime}\right)-x_{i}(\tilde{G})$ must hold for each $i \in N$.

Property 5: (Weak) unilateral disaggregation stability Lenzen et al. (2007) and Rodrigues and Domingos (2008) originally discussed the stability of pollution responsibility allocations due to de-merging of companies over supply chains. Let $G=(N, P, B, C, v)$ be our cooperative model. As a preliminary step, we formalize disaggregated supply chains with reference to the original model.

Let $P(i)\{j \in N \mid(i, j) \in P\}$ and $R(i)\{j \in N \mid(j, i) \in P\}$ be the subsets including firms, who are supplied by $i$ or supply firm $i$, respectively. Similarly, let $P_{i}$ and $R_{i}$ be the subsets including processes in which firm $i$ is a supplier or firm $i$ is supplied by, respectively. Assume that firm $i$ disaggregates its activities into two companies $i_{1}$ and $i_{2}$. After disaggregation, the set of firms is modified as $N^{\prime}=\left\{i_{1}, i_{2}\right\} \cup N \backslash\{i\}$. Then, the new set of processes is composed by $P^{\prime}=R(i) \times\left\{i_{1}\right\} \cup\left\{i_{2}\right\} \times P(i) \cup\left(i_{1}, i_{2}\right) \cup P \backslash\left(P_{i} \cup R_{i}\right)$. The new set of environmental costs $C^{\prime}$ is composed as it follows. First of all, we define $c_{j i_{1}}^{\prime}:=c_{j i}$ for $j \in R(i)$ and $c_{i_{2} j}^{\prime}, c_{i_{1} i_{2}}^{\prime} \geq 0$ where $c_{i j}:=c_{i_{2} j}^{\prime}+c_{i_{1} i_{2}}^{\prime}$ for each $j \in P(i)$. The last equality is named cost-splitting property. For the remaining cases, we simply require that $c_{h k}^{\prime}:=c_{h k}$. It is straightforward to think that disaggregated responsibility situations need a new responsibility matrix, that is $B^{\prime}$. First of all, such a new responsibility matrix $B^{\prime}$ must have $|\mathrm{N}|+1$ rows, that is the new number of firms, and $|\mathrm{P}|+1$ columns, that is the new number of processes. Such a new responsibility matrix 
might be defined in several ways. We require some reasonable assumption on it. Let $h$ be a firm participating in the original game and in the disaggregated game. Then it is immediate to see that $h$ cannot be firms $i_{1}, i_{2}, i$. For such a firm, we require some consistency property related to the original responsibility matrix $B$. Firstly, $B_{h p}=1$ with $p=(\mathrm{i}, \mathrm{j})$ implies $B_{\mathrm{h} p^{\prime}}^{\prime}=1$ with $p^{\prime}=\left(i_{2}, \mathrm{j}\right) ; B_{h p}=1$ with $p=\left(\mathrm{j}\right.$, i) implies $B_{\mathrm{h} p^{\prime}}^{\prime}=1$ with $p^{\prime}=\left(j, i_{1}\right)$. Finally $B_{\mathrm{h} p}^{\prime}=B_{\mathrm{h} p}$ holds if the process $p$ does not contain $i_{1}, i_{2}$.

The above conditions ensure that, if a firm is responsible for processes involving the firm $i$ in the original game, is also responsible for associated processes involving $i_{1}, i_{2}$ after the disaggregation. The latter means that the disaggregation does not create externalities on others' responsibilities for processes. In addition, we require that $\left(i_{1}, i_{2}\right) \notin B_{i_{1}}^{\prime} \cap B_{i_{2}}^{\prime}$; this means that, at most, one of the new companies is responsible for the internal process $\left(i_{1}, i_{2}\right)$, due to the disaggregation. If $B^{\prime}$ satisfies both conditions we say that a responsibility matrix $B^{\prime}$ is $B$-compatible.

We have defined disaggregated models $G^{\prime}=\left(N^{\prime}, P^{\prime}, B^{\prime}, C^{\prime}, v\right)$ from the original model $\mathrm{G}=(N, P, B, C, v)$. The multiplicity of disaggregated models depends on the multiple ways to split costs and/or on the multiple ways to implement new pollution responsibilities over supply chains.

Within this context, we say that $\mathrm{G}=(N, P, B, C, v)$ satisfies the unilateral disaggregation stability property if there exists a $B$-compatible responsibility matrix $B^{\prime}$ and, then, there exists a firm $i$ such that $x_{i_{1}}\left(G^{\prime}\right)+x_{i_{2}}\left(G^{\prime}\right)>x_{i}(G)$ for any disaggregated model $G^{\prime}=\left(N^{\prime}, P^{\prime}, B^{\prime}, C^{\prime}, v\right)$ (Unilateral Disaggregation Stability). We say that firm $i$ is the aggregating company and $B^{\prime}$ is the aggregating responsibility matrix. We say that $G$ satisfies weak unilateral disaggregation stability if $x_{j}(G) \geq x_{j}\left(G^{\prime}\right)$ (Weak Unilateral Disaggregation Stability) for each company $j$ different from $i$.

Assume that cost allocations satisfy unilateral disaggregation stability. In virtue of the cost-splitting property, it is straightforward to prove that:

$$
\sum_{h=1}^{n} \sum_{k=1}^{n} c_{h k} \geq \sum_{h=1}^{n+1} \sum_{k=1}^{n+1} c_{h k}^{\prime}
$$

Since cost allocations are efficient, we have

$$
\sum_{h=1}^{n} x_{h}(G)=\sum_{h=1}^{n} \sum_{k=1}^{n} c_{h k}=\sum_{h=1}^{n+1} x_{h}\left(G^{\prime}\right)=\sum_{h=1}^{n+1} \sum_{k=1}^{n+1} c_{h k}^{\prime} .
$$

We say that firm $i$ is the aggregating company. It follows that

$$
\sum_{h=1}^{n} x_{h}(G)-x_{\mathrm{i}}(G)>\sum_{h=1}^{n+1} x_{h}\left(G^{\prime}\right)-x_{i_{1}}\left(G^{\prime}\right)-x_{i_{2}}\left(G^{\prime}\right)
$$

The last formula implies that $x_{h}(G)>x_{h}\left(G^{\prime}\right)$ for some companies $h$. This means that there exists, at least, one firm who might consider the disaggregation option as a profitable one. If each company can get a benefit from disaggregation, then the whole supply chain can be considered as weakly stable, even if the aggregating firm prefers not to disaggregate its activities. In addition, if the cost of original game is strictly superior to the cost of the disaggregated game, there are internal incentives along supply chains to cause disaggregation. Even if cost allocations satisfy the unilateral disaggregation stability property, internal resources might still provide incentives to companies, which do not directly demerge, to collude and cause the disaggregation of the aggregating firm. 


\subsection{Additional remarks}

The first two properties (Equivalence and Equal Sharing of Extra Pollution) respect fairness by definition. It must be highlighted that, according to an egalitarianism principle, each company across a supply chain is granted the same right to pollute (Zhou and Wang 2016). As such, the total pollution across a supply chain might be increased due to unilateral actions from individual partners. However, in this case, an egalitarian perspective does not necessarily imply distributive justice concerning pollution responsibility allocation deriving from extra pollution (Rose 1990). Within this context, the equal sharing of extra pollution, no free riding and process independence properties provide additional commitment to substantive distributive justice.

\section{Linear supply chains and pollution responsibility}

Here, we specify our model $(N, P, B, C, v)$ in (2) where $v$ has been already defined in (1). The specifications deal with the structure of the process set $P$ and of the responsibility matrices $B$. A generic process $(i, j)$ represents the production of goods by company $i$ and the supply of such goods to company $j$. In the linear supply chain, the set $P_{i}$ is reduced to a singleton for each company, at most. This implies that company $i$ is supplying goods or services to a single supply chain partner. Then a process $(i, j)$ can be identified as an element of the set of companies. In addition, each process has an environmental cost $c_{i, i+1}$, which can be simply expressed by $c_{i}$. In the context, we can assume that existence of a final company $|\mathrm{N}|+1$, which is the company such that $P_{|N|+1}=\emptyset$. From company $\mid \mathrm{Nl}$, a process starts and supplies company $|\mathrm{N}|+1$. We assume that the final company has no responsibility, that is characterized by the following assumption $B_{|N|+1}=\emptyset$. Because allocations are responsible compatible by our initial choice, we know that $x_{|N|+1}=0$. Then, we disregard the last company $|N|+1$ because its cost allocation is null. Therefore the number of processes $|P|$ is equal to the number of pertinent firms is $|N|=n$.

Here, we define how the responsibility matrix $B$ is characterized according to environmental supply chain philosophies. In this context, three principles can be employed to solve the allocation problem. A Local Responsibility principle (LR), according to which each company $i$ is strictly responsible for the pollution costs, related to the production activities strictly happening at its premises. An Upstream Responsibility principle (UR), stating that upstream suppliers (dealing with raw material extraction, sub-component manufacturing and other energy intensive activities) are responsible not only for pollution happening at their premises, but can also influence the environmental performance of downstream partners. A Downstream Responsibility principle (DR), stating that downstream partners in the supply chain are responsible for the polluting activities happening at upstream suppliers' premises.

The three above principles can be exemplified by introducing the following responsibility matrices $B$. The following diagonal unitary $n \times n$ matrix characterizes LR:

$$
B=\left(\begin{array}{cccc}
1 & 0 & \ldots & 0 \\
0 & 1 & \ldots & 0 \\
\ldots & \ldots & \ldots & \ldots \\
0 & 0 & \ldots & 1
\end{array}\right)
$$


The UR principle says that player $j$ has the responsibility to participate in pollution mitigation expenses of each downstream supply chain partner. Then, the following unitary upper triangular $n \times n$ responsibility matrix characterizes the UR principle.

$$
B=\left(\begin{array}{cccc}
1 & 1 & \ldots & 1 \\
0 & 1 & \ldots & 1 \\
\ldots & \ldots & \ldots & \ldots \\
0 & 0 & \ldots & 1
\end{array}\right)
$$

Similarly, the following unitary lower triangular $n \times n$ responsibility matrix characterizes DR principle.

$$
B=\left(\begin{array}{cccc}
1 & 0 & \ldots & 0 \\
1 & 1 & \ldots & 0 \\
\ldots & \ldots & \ldots & \ldots \\
1 & 1 & \ldots & 1
\end{array}\right)
$$

After having defined the structure of process set $\mathrm{P}$ and the responsibility matrices $B$, the characteristic function, which has been defined in (1), depends just on the set of environmental costs, that is $C$. It will assume different forms for each of the three principles. By adopting the LR principle, the characteristic function (1) turns out to be:

$$
v_{1}(S)=\sum_{i \in S} c_{i}
$$

Conversely, it will assume the following form if a UR principle is adopted:

$$
\nu_{2}(S)=\sum_{i=j_{\min }}^{n} c_{i}, \quad j_{\min }=\min S .
$$

Finally, by adopting a DR principle, the characteristic function will take the following form:

$$
\nu_{3}(S)=\sum_{i=1}^{j_{\max }} c_{i}, \quad j_{\max }=\max S .
$$

Therefore the model $(N, P, B, C, v)$ in (2) can be simplified as $(N, C, v)$, according to the shape of the above-mentioned characteristic functions. In the following section, an allocation rule for the environmental responsibility costs is illustrated. This allocation rule is efficient, in such a way to ensure that $\sum_{i=1}^{n} x_{i}=\sum_{i=1}^{n} c_{i}$ among all the supply chain partners for all the three games introduced above $\left(N, C, v_{i}\right)$.

\section{The Shapley allocation for linear supply chains}

Among the possible allocation rules that can be derived from cooperative game theory, the Shapley value enjoys desirable properties such as efficiency and fairness (Owen 1995). The game $\left(N, C, v_{1}\right)$ admits a Shapley value $\mathrm{x}_{\mathrm{i}}=c_{i}$. The formula can be explicitly expressed by the following equations:

$$
\begin{aligned}
& x_{1}=c_{1} \\
& x_{2}=c_{2} \\
& x_{3}=c_{3} \\
& \cdots=\cdots \\
& x_{n}=c_{n}=0
\end{aligned}
$$


This particular allocation can be called Local Responsibility Sharing (LRS). For an arbitrary company $i$, if $c_{i}=0$ then $x_{i}=0$. In this case, LRS allocation is null for firm $i$, even if $B_{i} \neq \emptyset$. Mathematical results provided in Dong et al. (2012) are helpful to have a closedformula for the Shapley value for the game $\left(N, C, v_{2}\right)$. According to such results, $\left(N, C, v_{2}\right)$ admits a Shapley value which is equivalent to $x_{i}=\sum_{s=i}^{n} \mathrm{c}_{\mathrm{s}} / s$. The formula can be explicitly expressed by the following equations:

$$
\begin{aligned}
& x_{1}=c_{1}+\frac{c_{2}}{2}+\frac{c_{3}}{3}+\cdots+\frac{c_{n}}{n}=c_{1}+\frac{c_{2}}{2}+\frac{c_{3}}{3}+\cdots+\frac{c_{n-1}}{n-1} \\
& x_{2}=\frac{c_{2}}{2}+\frac{c_{3}}{3}+\cdots+\frac{c_{n}}{n}=\frac{c_{2}}{2}+\frac{c_{3}}{3}+\cdots+\frac{c_{n-1}}{n-1} \\
& x_{3}=\frac{c_{3}}{3}+\cdots+\frac{c_{n}}{n}=\frac{c_{3}}{3}+\cdots+\frac{c_{n-1}}{n-1} \\
& \cdots=\cdots \\
& x_{n}=\frac{c_{n}}{n}=0
\end{aligned}
$$

This particular allocation can be called Upstream Equal Sharing (UES) cost allocation. Similarly, thanks to the result provided in Ni and Wang (2007) and Gómez-Rúa (2013), the game $\left(N, C, v_{3}\right)$ admits a Shapley value, i.e. $\mathrm{x}_{\mathrm{i}}=\sum_{\mathrm{s}=1}^{\mathrm{i}} \mathrm{c}_{\mathrm{s}} /(\mathrm{n}-\mathrm{s}+1)$. The formula can be explicitly expressed by the following equations:

$$
\begin{aligned}
& x_{1}=\frac{c_{1}}{n} \\
& x_{2}=\frac{c_{1}}{n}+\frac{c_{2}}{n-1} \\
& x_{3}=\frac{c_{1}}{n}+\frac{c_{2}}{n-1}+\frac{c_{3}}{n-2} \\
& \cdots=\cdots \\
& x_{n}=\frac{c_{1}}{n}+\frac{c_{2}}{n-1}+\frac{c_{3}}{n-2}+\cdots+c_{n}=\frac{c_{1}}{n}+\frac{c_{2}}{n-1}+\frac{c_{3}}{n-2}+\cdots \frac{c_{n-1}}{n-1}
\end{aligned}
$$

This particular allocation can be called Downstream Equal Sharing (DES) allocation method. Here, we verify if LRS, DES and UES allocation rules satisfy the desirable properties introduced in Sect. 3.

Proposition 1 LRS, DES and UES allocation rules satisfy the equivalence property.

Proposition 2 LRS, DES and UES allocation rules satisfy the equal sharing of extra pollution property.

Proposition 3 LRS, DES and UES allocation rules satisfy the no free riding property.

Proposition 4 LRS, DES, UES cost allocations satisfy the process independence property.

Because the Shapley value is an efficient allocation, then UES cost allocations are efficient.

Proposition 5 LRS allocation rule does not satisfy the unilateral disaggregation stability property. We assume that $c_{n-1} \neq 0$ and $n>2$. Then, UES allocation rule satisfies unilateral disaggregation stability property. UES allocation rule satisfies weak unilateral disaggregation stability.

\section{Applications}

In order to demonstrate the applicability of the Shapley allocation (and related allocation rules) to real-world problems, the developed approach has been tested on an example related 
to the dairy supply chain. Dairy supply chains enjoy a very linear structure, in which the following stakeholders can be identified (Vergé et al. 2013):

- Feed producer, producing animal feed from raw agricultural products;

- Milk Producer, managing lactating animals (generally cows) and obtaining raw milk;

- Processor, performing important operations for converting milk into a product fit for human consumption (heat treatments and pasteurization) and transforming it into a final product to be sold onto the market (also taking care of the packaging element);

- Distributor, taking care of the logistical processes throughout the supply chain, involving cold chain operations;

- Retailer, making the product available to the final consumer.

- Consumers, buying and consuming the final product.

In a typical dairy supply chain, environmental pollution can be characterized through carbon equivalent emissions (expressed in $\mathrm{Kg} \mathrm{CO}_{2}$-eq per Litre). Combining findings from several sources (Vergé et al. 2013; Thoma et al. 2013; Ormond and Goodman 2015), environmental impacts (measured in terms of $\mathrm{Kg} \mathrm{CO}_{2}$-eq per litre of product) happening at each stage of the supply chain can be reported as shown in Table 1. For sake of simplicity, pollution costs might be deemed proportional to these environmental impacts.

The results of the three allocation principles shown in Sects. 3 and 4 (LRS, UES, DES) to a typical dairy supply chain are shown in the following Table 2 . It can be seen how the three allocation principles provide very different results, allocating different quota of environmental impacts to different actors. Under the LRS rule, the highest proportion of environmental impacts (and, therefore, of associated mitigation costs) is assigned to the Milk Producer, respecting a simple proportionality mechanism. UES and DES rules develop more complex mechanisms; notably, the DES rule strongly penalizes the Retailer and the Consumer.

It must be pointed out that the proposed allocation rules might be considered not mutually exclusive; indeed, convex combinations of these rules might be developed, as shown in Table 3, where LRS and DES are combined together. This could be done, for instance, in order to introduce, within a LRS framework, elements of downstream responsibility.

\section{Conclusions and future works}

This study shed light on normative frameworks for the problem of pollution responsibility allocation across multi-tier supply chains through cooperative game theory concepts. After a thorough literature review, the paper has presented a cooperative game theoretical responsi-

Table 1 Environmental impacts across a typical dairy supply chain

\begin{tabular}{ll}
\hline & Emissions $\left(\mathrm{Kg} \mathrm{CO}_{2}\right.$-eq per Litre $)$ \\
\hline Feed producer & 0.284 \\
Milk producer & 0.727 \\
Processor & 0.129 \\
Distributor & 0.108 \\
Retailer & 0.091 \\
Final consumer & 0.000 \\
Total & 1.337 \\
\hline
\end{tabular}


Table 2 Environmental impact allocation according to LRS, UES, DES rules

\begin{tabular}{lcrrr}
\hline Stakeholder & $\begin{array}{l}\text { Emissions share } \\
(\%)\end{array}$ & LRS $(\%)$ & DES (\%) & UES (\%) \\
\hline Feed producer & 21.21 & 21.24 & 3.54 & 55.03 \\
Milk producer & 54.34 & 54.38 & 14.42 & 33.78 \\
Processor & 9.61 & 9.65 & 16.83 & 6.60 \\
Distributor & 8.05 & 8.08 & 19.52 & 3.38 \\
Retailer & 6.79 & 6.81 & 22.92 & 1.36 \\
Consumers & 0.00 & 0.00 & 22.92 & 0.00 \\
Total & 100.00 & 100.00 & 100.00 & 100.00 \\
\hline
\end{tabular}

Table 3 Hybrid Allocation Rules

\begin{tabular}{lllc}
\hline Stakeholder & Emissions share (\%) & LRS +DES (50-50) (\%) & LRS + DES (80-20) (\%) \\
\hline Feed producer & 21.21 & 12.39 & 17.70 \\
Milk producer & 54.34 & 34.40 & 46.38 \\
Processor & 9.61 & 13.24 & 11.08 \\
Distributor & 8.05 & 13.80 & 10.37 \\
Retailer & 6.79 & 14.86 & 10.03 \\
Consumers & 0 & 11.46 & 4.58 \\
\hline
\end{tabular}

bility model for pollution allocation; the paper has detailed the generic model in the case of a linear supply chain, by selecting three different responsibility principles (namely: Upstream, Downstream and Local Responsibility) and developing some associated pollution responsibility allocation rules. These rules have been tested against some desirable properties that have been proposed. Furthermore, a practical application of the introduced cost allocation rules has been provided in the context of dairy supply chains.

The work proposed in this paper can be further extended in future researches, in order to overcome some of the limitations that characterize the current approaches. First of all, the model has been implemented on a simple linear supply chain, in which each company supplies and receives goods from a single partner; future work could extend the framework to more complex supply networks, in which multiple companies operate at each tier. Also, different pollution responsibility schemes and different supply chains structures (including, for instance, reverse elements, such as theorized by Jacobs and Subramanian 2012) might be employed in our model. A new set of normative properties could be defined, with the specific aim to assess the stability of international supply chains, especially against pollution costs differently perceived and taxed in nonhomogeneous countries.

Acknowledgements This research was partially supported by the project "Promoting Sustainable Freight Transport in Urban Contexts: Policy and Decision-Making Approaches (ProSFeT)", funded by the H2020MSCA-RISE-2016 programme (Grant Number: 734909). The authors are extremely grateful to the two anonymous referees; their very useful observations helped us lifting the quality of our paper in a very significant way.

Open Access This article is distributed under the terms of the Creative Commons Attribution 4.0 International License (http://creativecommons.org/licenses/by/4.0/), which permits unrestricted use, distribution, and repro- 
duction in any medium, provided you give appropriate credit to the original author(s) and the source, provide a link to the Creative Commons license, and indicate if changes were made.

\section{Appendix}

\section{Proof of Proposition 1}

Let $C$ be the set of environmental costs. Let $i, j \in N$ be two players such that $B_{i}=B_{j}$. Firstly, we prove that the LRS allocation rule satisfies equivalence. We know that $B_{i}=\{i\}$, $B_{j}=\{j\}$ by definition of the LRS method. By hypothesis we have $i=j$. Therefore, the condition $x_{i}=x_{j}$ is trivially satisfied.

Secondly, we prove that the UES allocation rule satisfies equivalence. In order to introduce a contradiction, we assume that $i<j$. Then we have $b_{i, i}=1$. But $b_{j, i}=0$. Therefore, we have that $i \in B_{i} \backslash B_{j} \neq \emptyset$. Then we have $B_{i} \neq B_{j}$, but this is an absurd. By symmetry, we simply conclude that $i=j$. Then $x_{i}=x_{j}$ is trivially satisfied.

Thirdly, we prove that the DES method satisfies the equivalence property. Assume that $i<j$. Then, we have $b_{i, j}=0$. However, we have $b_{j, i}=1$ because $i$ is upstream to $j$. Therefore, $j \in B_{j} \backslash B_{i} \neq \emptyset$. Therefore, we have $B_{j} \neq B_{i}$. This is an absurd by hypothesis. By symmetric arguments we simply conclude that $i=j$. Therefore $x_{i}=x_{j}$ holds.

\section{Proof of Proposition 2}

Let $G=(N, P, B, C, v)$ and $G^{\prime}=\left(N, P, B, C^{\prime}, v\right)$ be the two associated cooperative models where $\mathrm{C}, \mathrm{C}^{\prime}$ be two vectors of environmental costs with $\mathrm{C} \geq \mathrm{C}^{\prime}$. We pick two processes $p, q$. We define $A_{p q}=\left\{k \in N \mid b_{p, k}=b_{q, k}=1, c_{k}^{\prime}>c_{k}\right\}$.

Firstly, we prove that LRS satisfies the equal sharing of extra pollution. Because the responsibility matrix is an identity one, then $p=q=k$. Therefore $x_{k}\left(G^{\prime}\right)-x_{k}(G)=$ $x_{k}\left(G^{\prime}\right)-x_{k}(G)$ is satisfied because $p=q$ coincides with $k$.

Secondly, we prove that UES method satisfies equal sharing of extra pollution. Since the responsibility matrix is upper triangular, then we have $k \geq p$ and $k \geq q$. Therefore we obtain the following equalities

$$
\begin{aligned}
& x_{p}\left(G^{\prime}\right)-x_{p}(G)=\sum_{s=p}^{n} \frac{c_{s}^{\prime}}{s}-\sum_{s=p}^{n} \frac{c_{s}}{s}=\sum_{k \in A_{p q}} \frac{c_{k}^{\prime}}{k}-\frac{c_{k}}{k} \\
& x_{q}\left(G^{\prime}\right)-x_{q}(G)=\sum_{s=q}^{n} \frac{c_{s}^{\prime}}{s}-\sum_{s=q}^{n} \frac{c_{s}}{s}=\sum_{k \in A_{p q}} \frac{c_{k}^{\prime}}{k}-\frac{c_{k}}{k} .
\end{aligned}
$$

the two quantities are clearly equal since $\min A_{p q} \geq \max \{p, q\}$.

Thirdly, we prove that DES satisfies equal sharing of extra pollution property. As the responsibility matrix is upper triangular, then we have $\max A_{p q} \geq \min \{p, q\}$. Then we obtain that the following two expressions

$$
\begin{aligned}
& x_{p}\left(G^{\prime}\right)-x_{p}(G)=\sum_{k \in A_{p q}} \frac{c_{k}}{n-k+1}-\frac{c_{k}^{\prime}}{n-k+1} \\
& x_{q}\left(G^{\prime}\right)-x_{q}(G)=\sum_{k \in A_{p q}} \frac{c_{k}}{n-k+1}-\frac{c_{k}^{\prime}}{n-k+1}
\end{aligned}
$$

are equal. 


\section{Proof of Proposition 3}

Let $G=(N, P, B, C, v)$ and $G^{\prime}=\left(N, P, B, C^{\prime}, v\right)$ be the two associated cooperative models where $\mathrm{C}, \mathrm{C}^{\prime}$ are the two sets of environmental costs with $\mathrm{C}^{\prime} \geq \mathrm{C}$. Let $i \in N$. Suppose that $c_{j}^{\prime}=c_{j}$ for $j$ such that $b_{i, j}=1$.

Firstly, we prove that the LRS method satisfies the no free riding property. As the responsibility matrix is the identity matrix, from $b_{i, j}=1$ it thus follows that $i=j$. Then, we obtain $x_{i}(G)=x_{j}(G)=c_{j}, x_{i}\left(G^{\prime}\right)=x_{j}\left(G^{\prime}\right)=c_{j}^{\prime}=c_{j}$ since $c_{j}^{\prime}=c_{j}$ as per initial assumptions. From the above inequalities it follows that $x_{i}\left(G^{\prime}\right)=x_{i}(G)$. The latter implies that the no free riding property is satisfied.

We prove that the UES method satisfies no free riding. Because the responsibility matrix is upper triangular, from $b_{i, j}=1$ it thus follows that $i \leq j$. From the above assumption of no free riding axiom, we know that $c_{j}=c_{j}^{\prime}$ for $s \geq i$. From the UES method formulae, that is $x_{i}(G)=\sum_{j=i}^{n} c_{j} / j$ and $x_{i}\left(G^{\prime}\right)=\sum_{j=i}^{n} c_{j}^{\prime} / j$, we imply that $x_{i}(G)=x_{i}\left(G^{\prime}\right)$. Then, the UES allocation rule satisfies the no free riding property.

Thirdly, we prove that DES satisfies the no free riding property. As the responsibility matrix is an upper triangular matrix, from $b_{i, j}=1$ it thus follows that $i \geq j$. From the above assumption, we assume $c_{j}=c_{j}^{\prime}$ if $1 \leq j \leq i$. From the following DES allocation rules

$$
x_{i}(G)=\sum_{j=1}^{i} \frac{c_{j}}{n-j+1} x_{i}\left(G^{\prime}\right)=\sum_{j=1}^{i} \frac{c_{j}^{\prime}}{n-j+1}
$$

we imply that $x_{i}(G)=x_{i}\left(G^{\prime}\right)$. Therefore the DES method satisfies the no free riding property.

\section{Proof of Proposition 4}

Let $C, C^{\prime}, \mathrm{C}, \tilde{C}^{\prime}$ be four cost vectors. Let $G=(N, P, B, C, v), G^{\prime}=\left(N, P, B, C^{\prime}, v\right)$, $\tilde{G}=(N, P, B, \tilde{C}, v)$ and $\tilde{G}^{\prime}=(N, P, B, \tilde{C}, v)$ be the four cooperative models associated to the cost structures $C, C^{\prime}, \tilde{C}, \tilde{C}^{\prime}$. We assume that $C^{\prime} \geq C, \tilde{C}^{\prime} \geq \tilde{C}$. In addition $c_{j}^{\prime}=c_{j}, \tilde{c}_{j}^{\prime}=\tilde{c}_{j}$ for $j \in N \backslash\{k\}$ and $\tilde{c}_{k}^{\prime}=c_{k}^{\prime}$ and $c_{k}=\tilde{c}_{k}$. Pick $j \neq k$.

We prove that LRS satisfies the process independence property. Then $x_{j}\left(G^{\prime}\right)-x_{j}(G)=$ $c_{j}^{\prime}-c_{j}=0$ and $x_{j}\left(\tilde{G}^{\prime}\right)-x_{j}(\tilde{G})=\tilde{c}_{j}^{\prime}-\tilde{c}_{j}=0$ by hypothesis. The equality $c_{k}^{\prime}-\tilde{c}_{k}^{\prime}=c_{k}-\tilde{c}_{k}$ or $c_{k}^{\prime}-c_{k}=\tilde{c}_{k}^{\prime}-\tilde{c}_{k}$ holds by hypotheses. It is straightforward to see that $x_{k}\left(G^{\prime}\right)-x_{k}(G)=$ $c_{k}^{\prime}-c_{k}$ and $x_{k}\left(\tilde{G}^{\prime}\right)-x_{k}(\tilde{G})=\tilde{c}_{k}^{\prime}-\tilde{c}_{k}$.

We prove that UES satisfies process independence. Pick $j \neq k$. From the definition of usual UES allocation rules, we can easily compute the following differences

$$
x_{j}\left(G^{\prime}\right)-x_{j}(G)=\sum_{s=j}^{n} \frac{c_{s}^{\prime}}{s}-\sum_{s=j}^{n} \frac{c_{s}}{s}, x_{j}\left(\tilde{G}^{\prime}\right)-x_{j}(\tilde{G})=\sum_{s=j}^{n} \frac{\tilde{c}_{s}^{\prime}}{s}-\sum_{s=j}^{n} \frac{\tilde{c}_{s}}{s} .
$$

We assume that $k<j$; then, the two above quantities are equal to 0 since $c_{s}^{\prime}=c_{s}$, $\tilde{c}_{s}^{\prime}=\tilde{c}_{s}$. We assume that $k \geq j$; then, each addend in the above quantities is equal to 0 made exception for the ones indexed by $k$. These two addends in the two different above sums are $\left(c_{k}^{\prime} / k\right)-\left(c_{k} / k\right)$ and $\left(\tilde{c}_{k}^{\prime} / k\right)-\left(\tilde{c}_{k} / k\right)$ and they are equal by initial hypothesis.

We leave to the reader the proof for the DES allocation rule, which can be obtained following a very similar procedure. 


\section{Proof of Proposition 5}

Let $G=(N, C, v)$ be the associated cooperative model. After the disaggregation of company $i$, let call $G^{\prime}$ the new game. The new set of environmental costs $C^{\prime}$ includes the following components: $c_{h}^{\prime}=c_{h}$ for $h \neq i_{1}, i_{2}$ and $=c_{i}=c_{i_{2}}^{\prime}+c_{i_{2}}^{\prime}=c_{i}^{\prime}+c_{i+1}^{\prime}$. The matrix $B^{\prime}$ is a square matrix with order $\mathrm{n}+1$. The matrix $B^{\prime}$ is an LR matrix if the original responsibility matrix is an LR matrix and $B^{\prime}$ is an UR matrix if the original responsibility matrix is an UR matrix. In both cases it is straightforward to prove that $B^{\prime}$ is $B$-compatible.

We prove that the LRS allocation rule does not satisfy disaggregation stability. Let $i$ be the firm who disaggregates. Firstly, $i_{1} \notin B_{i_{2}}^{\prime}=\{i+1\}$. By definition of LRS allocations, we have the following formulas $x_{i}(G)=c_{i}, x_{i_{1}}\left(G^{\prime}\right)=x_{\mathrm{i}}\left(G^{\prime}\right)=c_{\mathrm{i}}^{\prime}=c_{i_{1}}^{\prime}$ and $x_{i_{2}}\left(G^{\prime}\right)=x_{\mathrm{i}+1}\left(G^{\prime}\right)=$ $c_{i+1}^{\prime}=c_{i_{2}}^{\prime}$. By hypothesis, $c_{i}=c_{i_{1}}^{\prime}+c_{i_{2}}^{\prime}$; then, the inequality becomes $x_{i}(G)=x_{i_{1}}\left(G^{\prime}\right)+$ $x_{i_{2}}\left(G^{\prime}\right)$. Therefore, the LRS allocation rule does not satisfy the unilateral disaggregation stability property.

We prove that UES allocation rule satisfies the disaggregation stability. First, we have that $i_{1}=i \notin B_{i_{2}}=\{\mathrm{i}+1, i+2, \ldots, n\}$. The UES cost allocation for firm $i$, who disaggregates, is $x_{i}(G)=\sum_{s=i}^{n} \frac{c_{s}}{s}=\sum_{s=i+1}^{n} \frac{c_{s}}{s}+\frac{c_{i}}{i}$. UES cost allocations for firms $i_{1}$ and $i_{2}$ are

$$
x_{i_{1}}\left(G^{\prime}\right)=\sum_{s=i_{1}}^{n+1} \frac{c_{s}^{\prime}}{s}=\sum_{s=i}^{n+1} \frac{c_{s}^{\prime}}{s}=\sum_{s=i+1}^{n+1} \frac{c_{s}^{\prime}}{s}+\frac{c_{i}^{\prime}}{i}, x_{i_{2}}\left(c^{\prime}\right)=\sum_{s=i_{2}}^{n+1} \frac{c_{s}^{\prime}}{s}=\sum_{s=i+1}^{n+1} \frac{c_{s}^{\prime}}{s} .
$$

By simple computations we have

$$
\begin{aligned}
x_{i_{1}}\left(G^{\prime}\right)+x_{i_{2}}\left(G^{\prime}\right)-x_{i}(G) & =\sum_{s=i+2}^{n+1} \frac{2 c_{s}^{\prime}}{s}+\frac{2 c_{i+1}^{\prime}}{i+1}-\sum_{s=i+1}^{n} \frac{c_{s}}{s}+\frac{c_{i}^{\prime}}{i}-\frac{c_{i}}{i} \\
& =\sum_{s=i+1}^{n}\left(\frac{2 c_{s+1}^{\prime}}{s+1}-\frac{c_{s}}{s}\right)+\frac{2 c_{i+1}^{\prime}}{i+1}+\frac{c_{i}^{\prime}}{i}-\frac{c_{i}}{i} .
\end{aligned}
$$

Let us assume that $i \neq n, n-1$. We rewrite the generic addend of the first sum in the right hand side of (5) in the following way:

$$
\frac{2 c_{s+1}^{\prime}}{s+1}-\frac{c_{s}}{s}=\frac{s c_{s}-c_{s}}{s(s+1)}=\frac{(s-1) c_{s}}{s(s+1)} \geq 0
$$

since $c_{s+1}^{\prime}=c_{s}$ for $s \geq i+1 \leq n$. Then the first sum in the right hand side of (5) contains the addend $(n-2) c_{n-1} /((n-1) n)$ since $i+1 \leq n-1$. The last above quantity is strictly positive because $c_{n-1}>0$ and $\mathrm{n}>2$ by hypothesis. Therefore, the first term in the right hand side of (5) is strictly positive. After simple computations, the algebraic difference of the last three remaining addends in the right hand part of equality (5) can be reworked as follows:

$$
\begin{aligned}
\frac{c_{i}^{\prime}}{i} & +\frac{2 c_{i+1}^{\prime}}{i+1}-\frac{c_{i}}{i}=\frac{c_{i}^{\prime}}{i}+\frac{2 c_{i+1}^{\prime}}{i+1}-\frac{c_{i}^{\prime}+c_{i+1}^{\prime}}{i}=\frac{2 c_{i+1}^{\prime}}{i+1}-\frac{c_{i+1}^{\prime}}{i} \\
= & \frac{2 c_{i+1}^{\prime} i-c_{i+1}^{\prime}(i+1)}{(i+1) i}=\frac{c_{i+1}^{\prime} i-c_{i+1}^{\prime}}{(i+1) i}=\frac{c_{i+1}^{\prime}(i-1)}{(i+1) i} \geq 0
\end{aligned}
$$

Therefore we have $x_{i_{1}}\left(G^{\prime}\right)+x_{i_{2}}\left(G^{\prime}\right)>x_{i}(G)$. Then UES satisfies unilateral disaggregation stability. In addition, we prove UES allocation rule satisfies weak unilateral disaggregation stability. We, more significantly, focus on the firms $j$ such that $j<i$. Then, let us consider just $i>1$. If $i=1$ there are no firms located upstream to $i=1$. Therefore, 
we again assume $i_{1}=i$ and $i_{2}=i+1$. For such companies, we have the following cost allocations

$$
\begin{gathered}
x_{j}(G)=\sum_{s=j}^{n} \frac{c_{s}}{s}=\sum_{j \leq s}^{i-1} \frac{c_{s}}{s}+\frac{c_{i}}{i}+\sum_{i+1 \leq s}^{n-1} \frac{c_{s}}{s} \\
x_{j}\left(G^{\prime}\right)=\sum_{s=j}^{n+1} \frac{c_{s}^{\prime}}{s}=\sum_{j \leq s}^{i-1} \frac{c_{s}^{\prime}}{s}+\frac{c_{i_{1}}^{\prime}}{i}+\frac{c_{i_{2}}^{\prime}}{i+1}+\sum_{i+2 \leq s}^{n} \frac{c_{s}^{\prime}}{s}
\end{gathered}
$$

By hypothesis, $c_{s}^{\prime}=c_{s}$ if $j \leq s<i$ and $c_{s+1}^{\prime}=c_{s}$ if $i+1 \leq s \leq n$. By virtue of the previous equalities, it is straightforward to prove that the first addend in the right-hand side of formula (6) is equal to the first addend in the right-hand side of formula (7). If $i \neq n$, the third addend in the right-hand side of formula (6) is strictly greater than the fourth addend in the right-hand side of formula (7) since $c_{n-1}>0 .{ }^{3}$ It thus follows that $x_{j}(G)>x_{j}\left(G^{\prime}\right)$ if and only if $c_{i} / i \geq\left(c_{i}^{\prime} / i\right)+\left(c_{i+1}^{\prime} /(i+1)\right)$. By cost-splitting formula, i.e. $c_{i}=c_{i}^{\prime}+c_{i+1}^{\prime}$, then the inequality becomes $c_{i}^{\prime} / i+c_{i+1}^{\prime} / i \geq c_{i}^{\prime} / i+c_{i+1}^{\prime} /(i+1)$. The last inequality is true; it is a strict inequality if $c_{i+1}^{\prime} \neq 0$ or it turns to be an equality if $c_{i+1}^{\prime}=0$. We can conclude that $x_{j}(G)>x_{j}\left(G^{\prime}\right)$. Let us assume that $j>i$. Firstly, we have the following inequalities:

$$
x_{n}(G)=x_{n}\left(G^{\prime}\right)=0, x_{n-1}(G)=\frac{c_{n-1}}{n-1}>\frac{c_{n}}{n}=\frac{c_{n}^{\prime}}{n}=x_{n-1}\left(G^{\prime}\right)
$$

because $c_{n}>0$. So we focus our attention on firms, which are different from $n-1, n$. As usual, UES allocations are $x_{j}(G)=\sum_{s=j}^{n-1} c_{s} / s$ and $x_{j}\left(G^{\prime}\right)=\sum_{s=j+1}^{n} c_{s}^{\prime} / s$. The first sum contains the addend $c_{n-1} /(n-1)$ and the second sum contains the addend $c_{n-1} / n$. Therefore, it follows that $x_{j}(G)>x_{j}\left(G^{\prime}\right)$ for $j>i$ and $j \neq n-1, n$. UES allocation rules satisfy weak unilateral disaggregation stability.

\section{References}

Bastianoni, S., Pulselli, F. M., \& Tiezzi, E. (2004). The problem of assigning responsibility for greenhouse gas emissions. Ecological Economics, 49(3), 253-257.

Bosquet, B. (2000). Environmental tax reform: Does it work? A survey of the empirical evidence. Ecological Economics, 34(1), 19-32.

Chander, P., \& Tulkens, H. (1995). A core-theoretic solution for the design of cooperative agreements on transfrontier pollution. International Tax and Public Finance, 2(2), 279-293.

Chung, S. H., Weaver, R. D., \& Friesz, T. L. (2013). Strategic response to pollution taxes in supply chain networks: Dynamic, spatial, and organizational dimensions. European Journal of Operational Research, 231(2), 314-327.

Dong, B., Ni, D., \& Wang, Y. (2012). Sharing a polluted river network. Environmental \& Resource Economics, 53(3), 367-387.

Eyckmans, J., \& Tulkens, H. (2003). Simulating coalitionally stable burden sharing agreements for the climate change problem agreements for the climate change problem. Resources and Energy Economics, 25, 299-327.

Filar, J. A., \& Gaertner, P. S. (1997). A regional allocation of world CO2 emission reductions. Mathematical and Computer Simulation, 43, 269-275.

Germain, M., \& Steenberghe, V. V. (2003). Constraining equitable allocations of tradable $\mathrm{CO}_{2}$ emission quotas by acceptability. Environmental and Resources Economics, 26, 469-492.

Gómez-Rúa, M. (2013). Sharing a polluted river through environmental taxes. SERIEs, 4(2), 137-153.

3 In case $i=n$, the third addend in the right-hand side of formula (6) and the fourth addend in the right-hand side of formula (7) are simply null. 
Gopalakrishnan, S., Granot, D., Granot, F., Sosic, G., Cui, H. (2016). Allocation of greenhouse gas emissions in supply chains. Mimeo, The University of Chicago Booth Business School. Available online at: http://f aculty.chicagobooth.edu/workshops/omscience/pdf/Autumn\%202016/GREEN\%20r1.pdf. Accessed 19 June 2018.

Imura, H., \& Moriguchi, Y. (1995). Economic interdependence and eco-balance: Accounting for the flow of environmental loads associated with trade. In S. Murai (Ed.), Toward global planning of sustainable use of the earth. Amsterdam: Elsevier.

Jacobs, B. W., \& Subramanian, R. (2012). Sharing responsibility for product recovery across the supply chain. Production and Operations Management, 21(1), 85-100.

Korhonen, J. (2002). The dominant economics paradigm and corporate social responsibility. Corporate Social Responsibility and Environmental Management, 9, 67-80.

Lenzen, M., \& Murray, J. (2010). Conceptualising environmental responsibility. Ecological Economics, 70(2), 261-270.

Lenzen, M., Murray, J., Sack, F., \& Wiedmann, T. (2007). Shared producer and consumer responsibility-theory and practice. Ecological Economics, 61(1), 27-42.

Leontief, W., \& Ford, D. (1970). Environmental repercussions and the economic structure: An input-output approach. Review of Economics and Statistics, 52, 262-271.

Liao, Z. L., Zhu, X. L., \& Shi, J. O. (2015). Case study on initial allocation of Shanghai carbon emission trading based on Shapley value. Journal of Cleaner Production, 103(15), 338-344.

MacKenzie, I. A., Hanley, N., \& Kornienko, T. (2008). The optimal initial allocation of pollution permits: A relative performance approach. Environmental Resources and Economics, 39, 265-282.

MacKenzie, I. A., Hanley, N., \& Kornienko, T. (2009). Using contests to allocate pollution rights. Energy Policy, 37(7), 2798-2806.

Ni, D., \& Wang, Y. (2007). Sharing a polluted river. Games and Economic Behavior, 60(1), 176-186.

Ormond, J., \& Goodman, M. K. (2015). A new regime of carbon counting: The practices and politics of accounting for everyday carbon through $\mathrm{CO}_{2}$-e. Global Environmental Change, 34, 119-131.

Owen, G. (1995). Game theory (3rd ed.). San Diego: Academic.

Proops, J. L. R., Faber, M., \& Wagenhals, G. (1993). Reducing $\mathrm{CO}_{2}$ emissions. A comparative input-output study for Germany and the UK. Berlin: Springer.

Ren, J., Bian, Y. W., Xu, X. Y., \& He, P. (2015). Allocation of product-related carbon emission abatement target in a make-to-order supply chain. Computers \& Industrial Engineering, 80, 181-194.

Ridgley, M. A. (1996). Fair sharing of greenhouse gas burdens. Energy Policy, 24(6), 517-529.

Rodrigues, J., \& Domingos, T. (2008). Consumer and producer environmental responsibility: Comparing two approaches. Ecological Economics, 66, 533-546.

Rose, A. (1990). Reducing conflict in global warming policy: The potential of equity as a unifying principle. Energy Policy, 18, 927-935.

Subak, S. (1995). Methane embodied in the international trade of commodities. Global Environmental Change, 5(5), 433-446.

Thoma, G., Popp, J., Nutter, D., Shonnard, D., Ulrich, R., Matlock, M., et al. (2013). Greenhouse gas emissions from milk production and consumption in the United States: A cradle-to-grave life cycle assessment circa 2008. International Dairy Journal, 31, 3-14.

UN Global. (2011). The ten principles. United Nations Global Compact. Available online at: https://www.un globalcompact.org/what-is-gc/mission/principles. Accessed 19 June 2018.

Vergé, X. P. C., Maxime, D., Dyer, J. A., Desjardins, R. L., Arcand, Y., \& Vanderzaag, A. (2013). Carbon footprint of Canadian dairy products: Calculations and issues. Journal of Dairy Science, 96(9), 6091-6104.

Viguier, L., Vielle, M., Haurie, A., \& Bernard, A. (2006). A two-level computable equilibrium model to assess the strategic allocation of emission allowances within the European Union. Computers \& Operations Research, 3, 369-385.

Wyckoff, A. W., \& Roop, J. M. (1994). The embodiment of carbon in imports of manufactured products-implications for international agreements on greenhouse gas emissions. Energy Policy, 22, 187-194.

Zhou, P., \& Wang, M. (2016). Carbon dioxide emissions allocation: A review. Ecological Economics, 125, 47-59. 